\title{
Prophylactic corticosteroid to prevent pain flare in bone metastases treated by radiotherapy
}

\author{
Gustavo Arruda Viani, Juliana Fernandes Pavoni, Ligia Issa De Fendi \\ Faculdade de Medicina de Ribeirão Preto da Universidade de São Paulo (FMRP-USP), Ribeirão Preto, Brazil
}

\begin{abstract}
Background: The aim of this study was to evaluate the effectiveness of prophylactic corticosteroids to prevent pain flare (PF) in bone metastases treated with radiotherapy performing a meta-analysis of randomized clinical trials (RCT).

Materials and methods: RCTs were identified on Medline, Embase, the Cochrane Library, and the proceedings of annual meetings through June 2020. We followed the PRISMA and MOOSE guidelines. A meta-analysis was performed to assess if corticosteroids reduce the PF, pain progression, and the mean of days with PF compared with the placebo. A p-value $<0.05$ was considered significant.

Results: Three RCTs with a total of 713 patients treated were included. The corticosteroids reduced the occurrence of early PF $20.5 \%$ (51/248) versus 32\% (80/250) placebo, OR $=0.55$ (95\% Cl: $0.36-0.82, p=0.002)$. The mean days of PF were reduced to 1.6 days $(95 \% \mathrm{Cl}: 1.3-1.9, \mathrm{p}=0.0001)$. Prophylactic corticosteroids had more patients with no $\mathrm{PF}$ and no pain progression, $\mathrm{OR}=1.63$ (95\% Cl: 1.14-2.32, $p=0.007)$. No significant corticosteroids effect was observed for pain progression $(p=n s)$ and late PF occurrence $(p=n s)$.

Conclusion: Prophylactic corticosteroids reduced the incidence of early PF, the days with PF, resulting in a superior rate of patients with no PF and no pain progression, but with no significant benefit for reducing pain progression or late PF occurrence. Key words: bone metastases; radiotherapy; pain flare; corticosteroids; meta-analysis Rep Pract Oncol Radiother 2021;26(2):218-225
\end{abstract}

\section{Introduction}

Bone pain due to metastases is the most common symptom demanding treatment in oncological patients [1]. Beside the pain, bone metastases (BM) can cause spinal cord compression, pathological fractures, and hypercalcemia [2]. All these complications secondary to BM have a massive impact on the quality of life (QoL).

Radiotherapy (RT) has a long history in the treatment of bone pain due to BM [2]. Several randomized clinical trials and meta-analyses show a significant reduction in pain and metastasis-related bone events with RT administration [3]. However, after the onset of an RT course, some patients may develop a transitory worsening of bone pain [4]. This effect is denominated as PF. The estimated incidence rate of PF is around 40\% [5]. In general, the effect occurs within the first 5 days after day one of RT administration (88\%) and, frequently, it has a mean duration of 3 days $[6,7]$. The radiobiologic mechanism that explains the occurrence of PF after $\mathrm{RT}$ is associated with the cytotoxic effect in the tissue irradiated $[8,9]$. RT produces and triggers an inflammatory response in the bone target irradiated. The inflammatory response induced by

Address for correspondence: Gustavo Arruda Viani, Dr. Rubem Aloysio Moreira St. 155, 4021686, São Paulo, fax: 55-16-34021744, tel: 55-16-34026584; e-mail: gusviani@gmail.com 
RT increases pro-inflammatory cytokines and chemokines in the local tissue, which worsens bone pain transitionally [9]. The relationship between $\mathrm{RT}$ and inflammatory response in the bone tissue has actively supported the design of randomized clinical trials evaluating glucocorticoids to prevent PF after RT [5, 10, 11]. Recently, some randomized clinical trials have been published, demonstrating contradictory results about corticosteroids' efficacy in reducing the incidence of PF [12-14]. The differences in the sample size, corticosteroids dose, and treatment duration are considered some of the problems of the studies analyzed individually.

Meta-analysis is a valuable tool to solve medical literature problems, mainly related to sample size and insufficient power of studies to find statistical differences between the arms of randomized clinical trials [15].

Based on this scenario, we designed a meta-analysis of randomized clinical trials to provide a general overview of the outcomes of the prophylactic use of corticosteroids to prevent PF in bone metastases irradiated.

\section{Materials and methods}

We conducted a systematic review and meta-analysis according to the Preferred Reporting Items for Systematic Reviews and Meta-analysis (PRISMA) statement [16]. Two reviewers performed the research, selecting articles initially by title and abstract, and then read the full article.

A systematic search was conducted by two of the investigators in PubMed, the Cochrane Central Register of Controlled Trials, and Embase for studies assessing the treatment outcomes of prophylactic corticosteroids to prevent $\mathrm{PF}$ in painful bone metastases treated with radiotherapy: ("bone and bones" [MeSH Terms] OR ("bone" [All Fields] AND "bones" [All Fields])) OR "bone and bones" [All Fields]) OR "bone" [All Fields]) AND ("metastase" [All Fields]) OR "metastasis" [All Fields]) OR "neoplasm metastasis" [MeSH Terms]) OR ("neoplasm" [All Fields] AND "metastasis" [All Fields])) OR "neoplasm metastasis" [All Fields]) OR "metastases" [All Fields]) OR "metastasize" [All Fields]) OR "metastasized" [All Fields]) AND ("radiotherapy" [MeSH Terms] OR "radiotherapy" [All Fields]) OR "radiotherapies" [All Fields]) OR "radiotherapy" [MeSH Subheading]) OR "radiotherapy s" [All
Fields]) AND ("pain" [MeSH Terms] OR "pain" [All Fields]) AND ("flare" [All Fields] OR "flares" [All Fields]). The lists containing the articles and reviews were checked, and possible related articles were tracked to complement the electronic query. Searches were performed from January 2000 up to June 2020 and were limited to publications in any language.

\section{Study selection}

We included only randomized clinical trials that evaluated the treatment outcomes of prophylactic corticosteroids to prevent PF compared with placebo in painful bone metastases treated by radiotherapy. Retrospective, non-randomized prospective studies and case reports were excluded.

\section{Patients}

We included studies of patients with painful bone metastases from any histological subtype, submitted or not to previous treatment with the metastatic lesion located in any bone treated by RT, and who received corticosteroids as a preventive treatment of the PF.

\section{Intervention}

We evaluated the efficacy of corticosteroids as an intervention to prevent PF. Studies administrating oral or intravenous corticosteroids before and during the radiotherapy course were included. Studies using multiple or single radiotherapy schedules with any total dose were included. Studies using conformational radiotherapy, tomotherapy, intensity-modulated radiotherapy, or VMAT, or stereotactic body radiotherapy (SBRT) were allowed.

\section{Outcomes}

The primary endpoint of the meta-analysis was the incidence of PF. PF was considered a minimum of a two-point increase in the worst pain score for the treated site without reducing analgesic intake, or $>25 \%$ increase in analgesic intake based on daily oral morphine equivalence without reducing the worst pain score. We considered PF to be early when the event occurred between days $0-5$, or late when it started on 6-14 days. Pain progression was defined as either an increase in the worst pain score of two or more without analgesic decrease or an analgesic increase of $25 \%$ or more from baseline without reducing the worst pain score. 


\section{Clinical data}

Two reviewers independently selected data using a standardized method. The following information was collected: author, year, study design, number of patients, gender, bone location, histological subtype, RT dose and RT technique, dexamethasone dosage, $\mathrm{PF}$ rate, pain progression rate, and days of PF. Two reviewers were in charge of gathering all data for all studies using a standardized data extraction form. A third reviewer was used to solve different issues by consensus.

\section{Data synthesis and analysis}

The rates of events of each outcome were calculated using the Odds ratio with the $95 \%$ confidence interval. The comparison for continuous variables was performed by mean difference estimation.

The $\mathrm{I}^{2}$ statistic illustrates the percentage of divergence across studies that is due to heterogeneity rather than chance. An $\mathrm{I}^{2}$ value lower than 25\% was interpreted as a low level of heterogeneity. We used the random-effect model due to a relevant variation in studies' characteristics. Two distinct methods were used to examine and explain the diversity among different studies results: subgroup analyses and meta-regression. A p-value lower than 0.05 was considered significant in all analyses. The meta-analysis was performed using the Open Meta-Analyst free open software.

\section{Results}

We identified in our searches 1,980 studies reporting the occurrence of $\mathrm{PF}$ after RT in painful bone metastases. After applying the inclusion criteria, 1,964 studies were excluded. The published studies were excluded due to many reasons, as described in the flowchart in Figure 1. Therefore, we selected 3 studies, including 713 patients using prophylactic dexamethasone to prevent $\mathrm{PF}$ after RT in painful BM compared with placebo. All studies were RCTs, and all of them were published from 2014 to 2020. In general, the treatment groups were similar for age, sex, and histological type. The main difference between the RCTs was due to the tumor location included in the Youssef et al. In this study, the authors only included patients with spine metastases randomized to receive methylprednisolone or placebo (saline infusion). Table 1 summarizes the characteristics of the studies included in the present meta-analysis. The placebo was the control arm in the other two trials, with dexamethasone $8 \mathrm{mg} / 4-5$ days as a similar intervention arm. In Youssef et al., the intervention arm was intravenous methylprednisolone $(5 \mathrm{mg} / \mathrm{kg}$ ) the day before RT.

\section{Early PF rate}

Two studies with 498 patients reported early PF as an outcome. Pooling the early PF rates of studies, comparing dexamethasone versus placebo, the rate of early $\mathrm{PF}$ was $20.5 \%$ (51/248) versus $32 \%$ (80/250) placebo, resulting in an OR $=0.55(95 \%$ CI: $0.36-0.82, \mathrm{p}=0.002)$, with no heterogeneity, $\mathrm{I}^{2}=0 \%$ (Fig. 2A).

\section{Late PF}

Three RCTs with 611 patients reported late $\mathrm{PF}$ rate as an outcome. Pooling the late PF rate of RCTs, comparing dexamethasone versus placebo, the rate of late $\mathrm{PF}$ was $18.6 \%$ (56/301) versus $21.6 \%(67 / 310)$, providing an $\mathrm{OR}=0.74(95 \% \mathrm{CI}$ : 0.41-1.33), with no heterogeneity, $\mathrm{I}^{2}=0 \%$ (Fig. $2 \mathrm{~B}$ ).

\section{Mean days with PF}

Three studies, including 611 patients, reported the mean days with PF. Combining the studies and calculating the mean difference between corticosteroids and placebo arms, a significant difference was found of 1.94 days (95\% CI: -1.3 to -2.5 , $\mathrm{p}<0.0001$ ), with heterogeneity $\mathrm{I}^{2}=78 \%, \mathrm{p}=0.001$ ) (Fig. 3A). In the sensitivity analysis, we removed the Youssef study by exploring the heterogeneity due to the inclusion of only spinal metastases. After that, the two studies combined gave a mean difference in PF days of $-1.6(95 \% \mathrm{CI}-1.3$ to $-1.9, \mathrm{p}=0.0001)$, with no heterogeneity, $\mathrm{I}^{2}=16 \%, \mathrm{p}=0.27$, validating the outcome (Fig. 3B).

\section{No PF/no pain progression}

Two studies with 498 patients reported no PF/PP as an outcome. Pooling the no PF/PP of studies, comparing dexamethasone versus placebo, the rate of no $\mathrm{PF} / \mathrm{PP}$ was $52 \%$ (129/248) versus $40 \%$ (100/250) placebo, resulting in an $\mathrm{OR}=1.63(95 \%$ CI: $1.14-2.32, \mathrm{p}=0.007$ ), (Fig. 2), with no heterogeneity, $\mathrm{I}^{2}=0 \%$ (Fig. $4 \mathrm{~A}$ ).

\section{Pain progression}

Two studies reported PP as an outcome. Two studies with 498 patients reported PP as an out- 


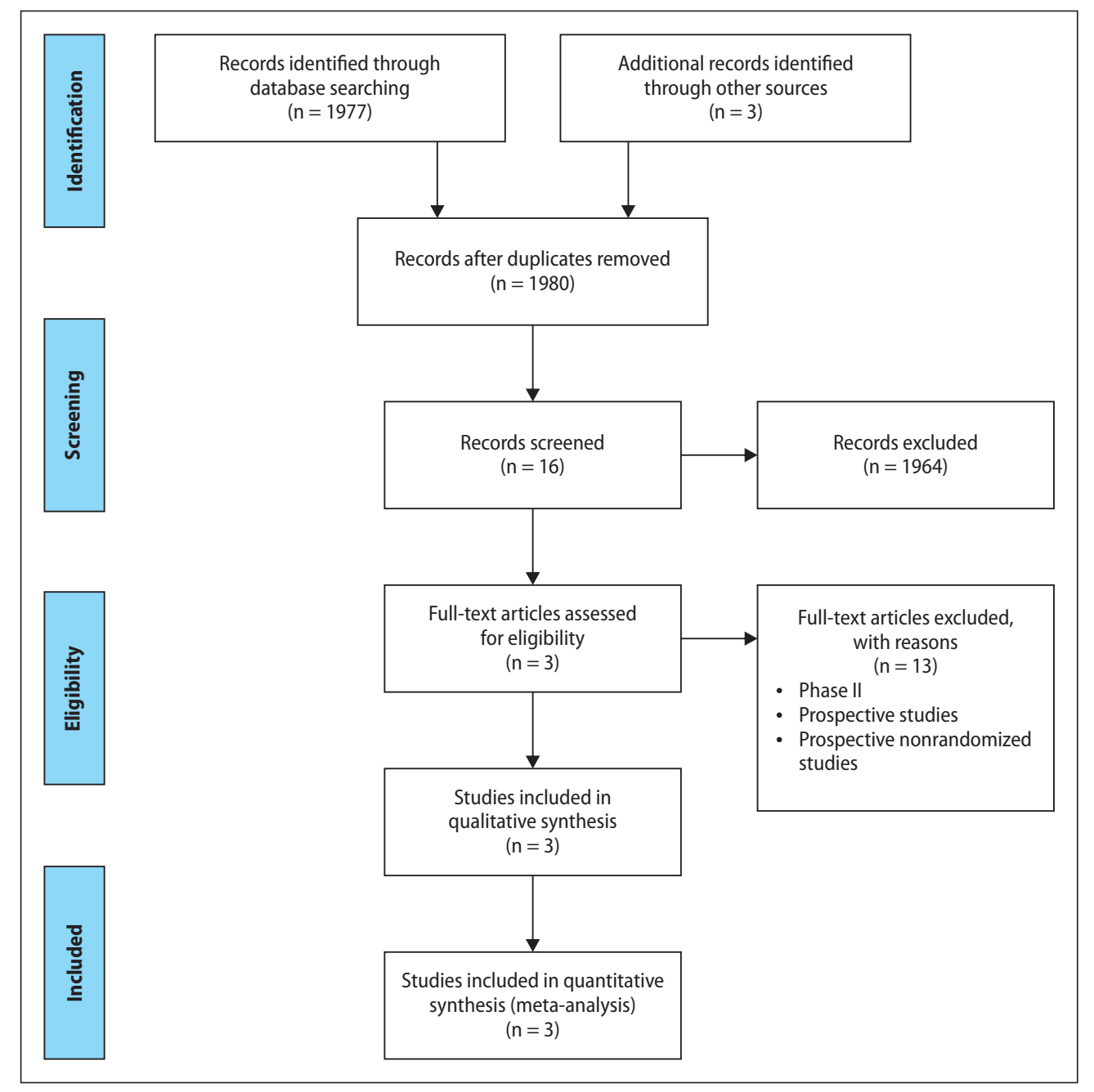

Figure 1. Flowchart of studies included in the meta-analysis

Table 1. Characteristics of the studies included in the meta-analysis

\begin{tabular}{|c|c|c|c|c|c|c|}
\hline \multirow{2}{*}{$\begin{array}{l}\text { Study } \\
\text { Variables }\end{array}$} & \multicolumn{2}{|c|}{ Chow et al. [14] } & \multicolumn{2}{|c|}{ Liden et al. [12] } & \multicolumn{2}{|c|}{ Youssef et al. [13] } \\
\hline & Corticosteroid & Placebo & Corticosteroid & Placebo & Corticosteroid & Placebo \\
\hline Patients & 148 & 150 & 100 & 100 & 60 & 60 \\
\hline Age (mean) & & & 67 & 68 & 65 & 63 \\
\hline Male (\%) & 59 & 55 & 62 & 62 & 58 & 55 \\
\hline $\begin{array}{l}\text { Histology (\%) } \\
\text { Lung } \\
\text { Breast } \\
\text { Prostate }\end{array}$ & $\begin{array}{l}28 \\
22 \\
24\end{array}$ & $\begin{array}{l}29 \\
22 \\
25\end{array}$ & $\begin{array}{l}17 \\
27 \\
30\end{array}$ & $\begin{array}{l}25 \\
23 \\
30\end{array}$ & $\begin{array}{c}36 \\
26 \\
7\end{array}$ & $\begin{array}{l}30 \\
23 \\
10\end{array}$ \\
\hline $\begin{array}{l}\text { Location } \\
\text { Spine } \\
\text { Other }\end{array}$ & $\begin{array}{l}30 \\
70\end{array}$ & $\begin{array}{l}40 \\
60\end{array}$ & & & 100 & 100 \\
\hline $\begin{array}{l}\text { RT fractionation (\%) } \\
\text { Single } \\
\text { Multiple }\end{array}$ & $\begin{array}{c}100 \\
0\end{array}$ & $\begin{array}{c}100 \\
0\end{array}$ & $\begin{array}{l}77 \\
23\end{array}$ & $\begin{array}{l}81 \\
19 \\
\end{array}$ & 100 & 100 \\
\hline Corticosteroid & Dexamethasone & & Dexamethasone & & Methylprednisolone & \\
\hline Corticosteroid dosage & $\begin{array}{l}8 \mathrm{mg} / \text { day } \\
\text { for } 5 \text { days }\end{array}$ & & $\begin{array}{l}8 \mathrm{mg} / \text { day } \\
\text { for } 4 \text { days }\end{array}$ & & $\begin{array}{c}5 \mathrm{mg} / \mathrm{kg} 1 \text { days } \\
\text { before RT }\end{array}$ & \\
\hline
\end{tabular}




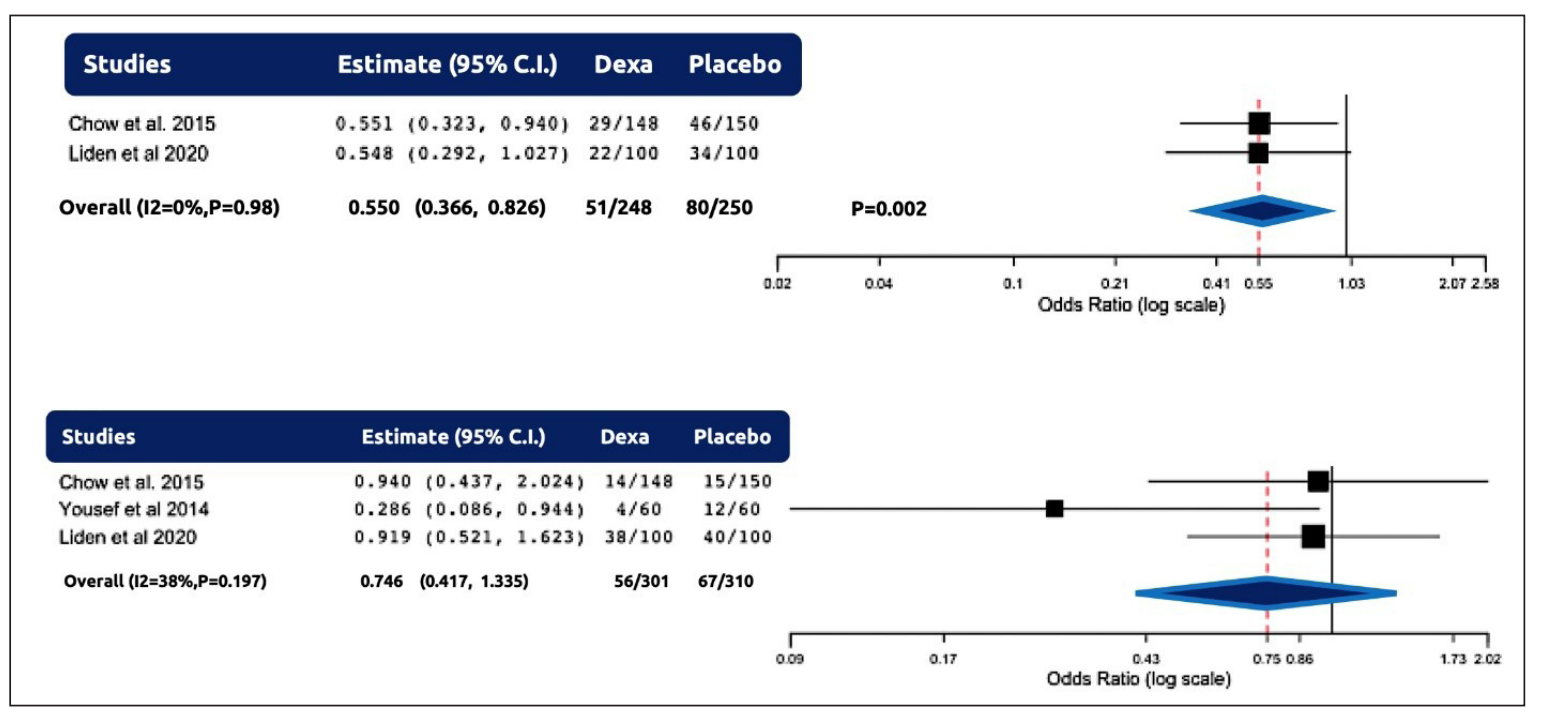

Figure 2. A. Early pain flare; B. Late pain flare

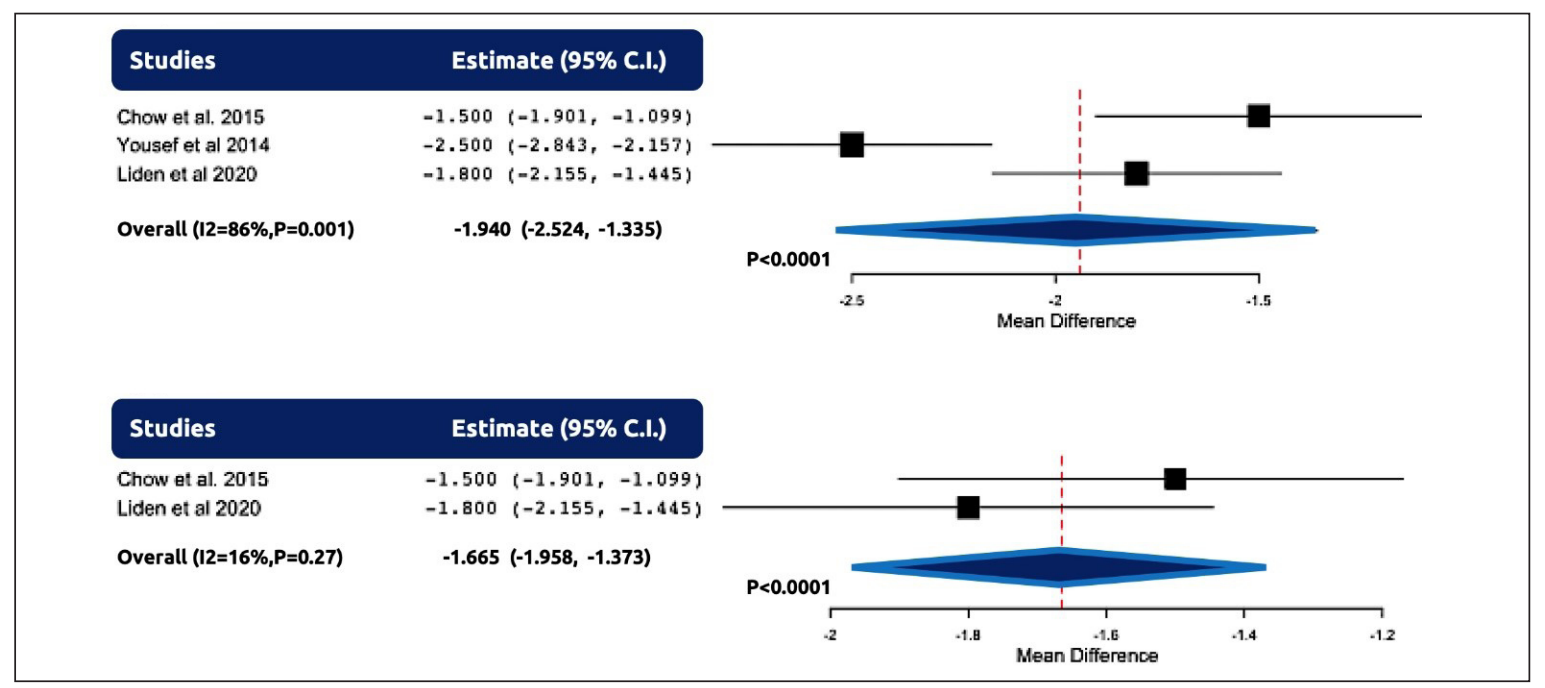

Figure 3. A. Mean of days with pain flare without sensitivity analysis; B. Mean of days with pain flare with sensitivity analysis

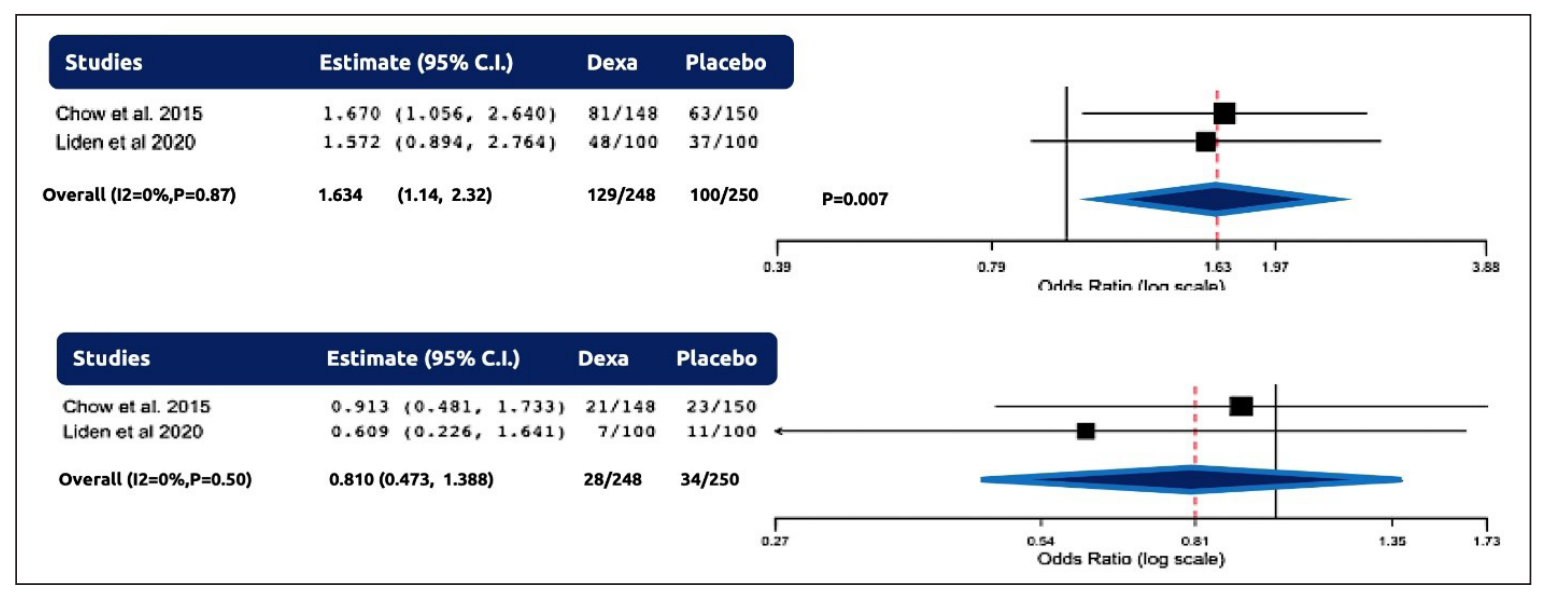

Figure 4. A. No pain flare and no pain progression; B. Pain progression 
come. Pooling the PP of studies, comparing dexamethasone versus placebo, the rate of $\mathrm{PP}$ was $11.2 \%$ $(28 / 248)$ versus $13.6 \%(34 / 250)$ placebo, resulting in an OR $=0.81$ (95\% CI: 0.4-1.3, p = NS) (Fig. 4B), with no heterogeneity, $\mathrm{I}^{2}=0 \%$.

\section{Discussion}

The present study is the first meta-analysis, including only randomized clinical trials, assessing the benefit of prophylactic corticosteroids administration to prevent $\mathrm{PF}$ in painful BM. We gathered a total of 3 RCTs to estimate the incidence of PF, $\mathrm{PP}$, and days of PF, comparing corticosteroids with placebo. In general, the heterogeneity for most of the outcomes evaluated was not significant, which validated our findings. We divided the analysis of $\mathrm{PF}$ into early and late, mainly to investigate if the corticosteroids effect is durable or transitory. The meta-analysis shows a significant effect of corticosteroid for PF occurrence in the first week of the initiation of radiotherapy. On the other hand, prophylactic treatment had no significant difference compared with the placebo for the late occurrence of PF. This finding raises the question if corticosteroids dosage or the short intake time (4-5 days) is ineffective in producing a long-lasting protective effect for the occurrence of PF. In both RCTs using oral dexamethasone, a dose of $8 \mathrm{mg} /$ day for $4-5$ days was used. The RCT conducted by Liden et al. had three arms with one of them using $8 \mathrm{mg}$ for 1 day followed by 3 days of placebo. The early PF rate in this arm was $73 \%$, with no significant difference for $8 \mathrm{mg}$ per 4-5 days (52\%) in the early evaluation. Although our study was not designed to evaluate the length of corticosteroids administration, these data make this question inevitable. The literature reports that the majority of PF occurs during the first week of RT $[4,7]$. However, our data shows that late PF was significant in the placebo arms in all RCTs (21.6\%), and a more extended treatment schedule with corticosteroids may be necessary to find a significant difference for this endpoint. The RCT conducted by Yousef et al. randomized 120 patients to receive a $24-\mathrm{h}$ infusion of methylprednisone $(5 \mathrm{mg} / \mathrm{kg})$ or placebo (normal saline infusion) the day before the initiation of radiotherapy. Four (7\%) patients in the intervention group and $12(20 \%)$ patients in the placebo group had PF $(\mathrm{p}<0.05)$. The dose of corticosteroids in their study was much higher than the doses used in the other two studies. If we convert the dose used by Youssef et al. $5 \mathrm{mg} / \mathrm{kg}$ would be equivalent to $65 \mathrm{mg} /$ day for a $70 \mathrm{~kg}$ patient. This brutal difference in corticosteroids dosage between Youssef et al. and other trials had a direct impact on the rate and duration of PF. The mean duration of PF in Youssef et al. was 125 days in the intervention group and 375 days in the placebo group. Combining the three trials, the mean of PF days was significant but with heterogeneity. When Youssef trial was excluded, the result remained significant and with no heterogeneity, which validates the outcomes. Another question arising from our data regards the sample size of the studies. The studies may be considered underpowered to detect the estimated difference from the statistical assumptions. For instance, Chow et al. [14] postulated a difference in the incidence of $17 \%$ between the arms. However, the group difference was $8.9 \%$ in the intention-to-treat, and the incidence of late PF was practically the same between placebo and dexamethasone (9.4\% and 10\%) [14]. Thus, even increasing the sample size, it is improbable to imagine a significant difference between the arms. Our meta-analysis clarifies this point, with data from 611 patients randomized with a difference of $3 \%$ between the treatment arms and no statistical significance was detected. The absence of the benefit in the corticosteroids arm of reducing late PF reveals the necessity of changing the treatment strategy. For us, the way to do that would be to increase the corticoid dose or increase administration time to reduce the occurrence of late PF significantly. One example of this strategy is the corticosteroids administration to avoid cranial hypertension during brain metastases radiotherapy course. Brain metastases typically are treated with the administration of $16 \mathrm{mg} /$ day of dexamethasone during 10 fractions of whole-brain radiotherapy without clinical severe adverse effects. Based on this experience, the changing of corticosteroids dose or intake time should not pose a significant challenge.

Pain progression was another endpoint evaluated in our meta-analysis, and no significant difference between intervention and placebo was observed. RT effectively reduces the pain of BM independently of the histological subtype and the radiotherapy schedule employed [3]. Although corticosteroids have co-analgesic properties, the effectiveness of RT in reducing pain is so significant that the addiction of corticosteroids provides no benefit for this out- 
come. On the other hand, the combination effectively maintained a high proportion of patients without $\mathrm{PF}$ and no pain progression than the placebo.

Although our study is a meta-analysis of RCTs, it has some limitations. The evaluation of some aspects with a possible relationship with $\mathrm{PF}$ occurrence in a meta-regression analysis was not explored, such as tumor histology, RT fractionation, bone location, and pain score baseline. We also did not evaluate the adverse effects of corticosteroids administration. However, in all the published RCTs the adverse effects had no significant differences between the study arms, which demotivated us to analyze this endpoint, and also none of the RCTs performed a subgroup analysis of these possible prognostic factors to provide information for performing a metaregression.

\section{Conclusion}

Prophylactic corticosteroids reduce significantly the occurrence of early PF in bone metastases treated with radiotherapy. It also reduces the mean of days with $\mathrm{PF}$ and maintains a significant patient rate with no $\mathrm{PF}$ and no pain progression. However, the corticosteroids did not affect the incidence of late PF. Although it has co-analgesic properties, its use combined with RT did not produce any difference in the pain progression rate compared with the placebo. Consequently, its use should be strictly recommended to reduce early $\mathrm{PF}$ and shorten the days with PF in bone metastases treated with RT. Our findings call attention for a change in the strategy to prevent $\mathrm{PF}$ in future randomized clinical trials, which should consider increasing the corticosteroids dose or the time of intake, establishing the late PF rate as the primary endpoint.

\section{Conflict of interest}

The authors declare that they have no competing interests.

\section{Funding}

The authors declare none funding to produce the research.

\section{Ethical statement}

The present study is a meta-analysis of published randomized clinical trials and the ethical committee approval was not necessary.

\section{References}

1. Løhre ET, Thronæs M, Klepstad P. Breakthrough cancer pain in 2020. Curr Opin Support Palliat Care. 2020; 14(2): 94-99, doi: 10.1097/SPC.0000000000000494, indexed in Pubmed: 32332210

2. Culleton S, Kwok S, Chow E. Radiotherapy for pain. Clin Oncol (R Coll Radiol). 2011; 23(6): 399-406, doi: 10.1016/j. clon.2010.11.011, indexed in Pubmed: 21168999.

3. Chow R, Hoskin P, Schild SE, et al. Single vs multiple fraction palliative radiation therapy for bone metastases: Cumulative meta-analysis. Radiother Oncol. 2019; 141: 56-61, doi: 10.1016/j.radonc.2019.06.037, indexed in Pubmed: 31445837.

4. Goldfinch R, White N. An investigation into the incidence of pain flare in patients undergoing radiotherapy for symptomatic bone metastases. Radiography (Lond). 2018; 24(3): 192-195, doi: 10.1016/j.radi.2018.01.010, indexed in Pubmed: 29976330.

5. McDonald R, Chow E, Rowbottom L, et al. Incidence of pain flare in radiation treatment of bone metastases: A literature review. J Bone Oncol. 2014; 3(3-4): 84-89, doi: 10.1016/j.jbo.2014.10.001, indexed in Pubmed: 26909303.

6. Gomez-Iturriaga A, Cacicedo J, Navarro A, et al. Incidence of pain flare following palliative radiotherapy for symptomatic bone metastases: multicenter prospective observational study. BMC Palliat Care. 2015; 14: 48, doi: 10.1186/ s12904-015-0045-8, indexed in Pubmed: 26427616.

7. Hird A, Chow E, Zhang L, et al. Determining the incidence of pain flare following palliative radiotherapy for symptomatic bone metastases: results from three canadian cancer centers. Int J Radiat Oncol Biol Phys. 2009; 75(1): 193-197, doi: 10.1016/j.ijrobp.2008.10.044, indexed in Pubmed: 19167840.

8. Loi M, Klass ND, De Vries KC, et al. Pain flare, complexity and analgesia in bone oligometastases treated with stereotactic body radiation therapy. Eur J Cancer Care (Engl). 2018; 27(6): e12915, doi: 10.1111/ecc.12915, indexed in Pubmed: 30246916.

9. MacLeod K, Laird BJA, Carragher NO, et al. Predicting Response to Radiotherapy in Cancer-Induced Bone Pain: Cytokines as a Potential Biomarker? Clin Oncol (R Coll Radiol). 2020; 32(10): e203-e208, doi: 10.1016/j.clon.2020.03.010, indexed in Pubmed: 32284199.

10. Lim FMY, Bobrowski A, Agarwal A, et al. Use of corticosteroids for pain control in cancer patients with bone metastases: a comprehensive literature review. Curr Opin Support Palliat Care. 2017; 11(2): 78-87, doi: 10.1097/ SPC.0000000000000263, indexed in Pubmed: 28306570.

11. Niglas M, Raman S, Rodin D, et al. Should dexamethasone be standard in the prophylaxis of pain flare after palliative radiotherapy for bone metastases?-a debate. Ann Palliat Med. 2018; 7(2): 279-283, doi: 10.21037/apm.2017.04.08, indexed in Pubmed: 28595438.

12. van der Linden YM, Westhoff PG, Stellato RK, et al. Dexamethasone for the Prevention of a Pain Flare After Palliative Radiation Therapy for Painful Bone Metastases: The Multicenter Double-Blind Placebo-Controlled 3-Armed Randomized Dutch DEXA Study. Int J Radiat Oncol Biol Phys. 2020; 108(3): 546-553, doi: 10.1016/j.ijrobp.2020.05.007, indexed in Pubmed: 32446951. 
13. Yousef AAM, El-Mashad NM. Pre-emptive value of methylprednisolone intravenous infusion in patients with vertebral metastases. A double-blind randomized study. J Pain Symptom Manage. 2014; 48(5): 762-769, doi: 10.1016/j.jpainsymman.2013.12.232, indexed in Pubmed: 24704801.

14. Chow E, Meyer RM, Ding K, et al. Dexamethasone in the prophylaxis of radiation-induced pain flare after palliative radiotherapy for bone metastases: a double-blind, randomised placebo-controlled, phase 3 trial. Lancet Oncol. 2015; 16(15): 1463-1472, doi:
10.1016/S1470-2045(15)00199-0, indexed in Pubmed: 26489389.

15. Fagard RH, Staessen JA, Thijs L. Advantages and disadvantages of the meta-analysis approach. J Hypertens Suppl. 1996; 14(2): S9-12; discussion S13, doi: 10.1097/00004872199609002-00004, indexed in Pubmed: 8934372.

16. Liberati A, Altman DG, Tetzlaff J, et al. The PRISMA statement for reporting systematic reviews and meta-analyses of studies that evaluate healthcare interventions: explanation and elaboration. BMJ. 2009; 339: b2700, doi: 10.1136/ bmj.b2700, indexed in Pubmed: 19622552. 